Onkologe 2018 $24: 921$

https://doi.org/10.1007/s00761-018-0465-1

Online publiziert: 18. September 2018

(c) Springer Medizin Verlag $\mathrm{GmbH}$, ein Teil von Springer Nature 2018

CrossMark

Rainer Ullmann

Hamburg, Deutschland

\title{
Leserbrief zu Steger et al. Methadon gegen Krebs
}

\section{Einige Aspekte fehlen}

Ethisch geprüft werden muss entsprechend, ob ein Medikament mit geringen UAW bei einer nicht heilbaren Krebserkrankung eingesetzt werden darf, wenn der Nutzen nicht wissenschaftlich bewiesen ist. Berücksichtigt werden muss bei dieser ethischen Prüfung, dass als Standardtherapie Medikamente mit manchmal schweren UAW eingesetzt werden, die zwar nicht heilen, aber statistisch gesichert die Überlebenszeit manchmal um einige Monate verlängern.

Die Autoren hätten weitere Fragen prüfen sollen. Seit 25 Jahren ist aus Zellkulturstudien verschiedener Arbeitsgruppen bekannt, dass einige Krebszellen Opioidrezeptoren exprimieren und verschiedene Opioide hemmend oder fördernd das Wachstum beeinflussen. Mit Methadon konnte in Zellkulturen das Wachstum von einigen Krebszellen gehemmt werden. Ist es ethisch vertretbar, die Zugabe von Methadon zur Standardchemotherapie nicht $\mathrm{zu}$ untersuchen, sondern die (im Vergleich zu Chemotherapeutika) geringen $\mathrm{Ne}$ benwirkungen dieses lange bekannten Opioids maßlos aufzubauschen?

Nach den Angaben einiger Patienten drohen Onkologen damit, die Chemotherapie abzubrechen, wenn die Patienten zusätzlich Methadon nehmen wollen: Ist das ethisch vertretbar?

Zur Fachautorität medizinischer Fachgesellschaften: Darauf konnte man sich bei der Einführung der Substitutionsbehandlung Heroinabhängiger nicht verlassen. Diese Behandlung wurde in den 1970er und 1980er Jahren von einflussreichen Psychiatern mit den abstrusesten, wissenschaftlich nicht begründeten Argumenten abgelehnt und ist jetzt Standardbehandlung. Eine kritischere Bewertung der Äußerungen von Fachgesellschaften erscheint sinnvoll.

\section{Korrespondenzadresse}

Dr. Rainer Ullmann

Ophagen 15, 20257 Hamburg, Deutschland r.ullmann@gmx.de

Interessenkonflikt. R. Ullmann gibt an, dass kein Interessenkonflikt besteht. 